Research Paper

\title{
The Importance Of Multi-site Intra-operative Tissue Sampling In The Diagnosis Of Hip And Knee Periprosthetic Joint Infection - Results From A Single Centre Study
}

\author{
Lucy C. Walker ${ }^{1}$, Nick D. Clement ${ }^{1}$, Ian Wilson ${ }^{2}$, Munawar Hashmi ${ }^{1}$, Julie Samuel ${ }^{1}$, David J. Deehan ${ }^{1}$ \\ 1. Freeman Hospital, Newcastle-upon-Tyne Hospitals NHS Foundation Trust, Freeman Road, Newcastle-upon-Tyne, NE7 7DN, UK \\ 2. Institute of Genetic Medicine, Newcastle University, International Centre for Life, Newcastle-upon-Tyne, NE7 7DN, UK \\ $\bowtie$ Corresponding author: 1.c.walker2@newcastle.ac.uk
}

(c) The author(s). This is an open access article distributed under the terms of the Creative Commons Attribution License (https://creativecommons.org/licenses/by/4.0/). See http://ivyspring.com/terms for full terms and conditions.

Received: 2019.08.20; Accepted: 2019.11.28; Published: 2020.05.18

\begin{abstract}
Introduction: The primary aim of this study was to determine whether the tissue type and anatomical location of intra-operative samples influences the accuracy of culture in the diagnosis of periprosthetic joint infection (PJI). The secondary aim was to create a predictive model of PJI using other known patient variables.

Methods: A retrospective cohort of 3460 intra-operative samples from 887 patients was identified. The data was then analysed to compare intra-operative culture results (positive or negative) to the chosen gold standard of clinical diagnosis made by the treating team (infected or non-infected prosthetic joint). The intra-operative samples were grouped according to their labelling at the time of collection.

Results: No single tissue type or anatomical location had both high sensitivity and specificity. The highest specificity for an anatomical location was hip bursa with $100 \%$, for tissue type it was synovium with $93 \%$. Sensitivity was highest in the anatomical locations for hip capsule (68\%) and in the tissue types for pus (83\%). Data analysis was performed to create a model for PJI and identified pre-operative predictors of PJI (increased white cell count, knee joint and non-revision surgery) which when used in combination with intra-operative culture results increased the sensitivity.

Conclusion: Sample type and anatomical location influenced the reliability of the diagnosis of PJI however, no single sample type had higher diagnostic accuracy than samples combined thereby highlighting the necessity of obtaining multiple intra-operative samples in the diagnosis of PJI. The variation in predictive values of tissue types as well as improvement in sensitivity when combined with patient factors indicates that types of intra-operative sampling and the overall diagnostic pathway should vary depending on the individual case.
\end{abstract}

Key words: Periprosthetic joint infection; intra-operative samples; culture; predictive model

\section{Introduction}

Periprosthetic joint infection (PJI) is the second most common complication of joint arthroplasty [1], with a reported occurrence of $0.94 \%$ of primary total hip and knee arthroplasties in the UK [2]. Culture results are considered extremely useful as they not only provide a diagnosis but also identify the specific pathogens and sensitivity profiles [3-5]. An incorrect diagnosis of PJI may lead to an unnecessary surgical procedure [6] whereas not recognising PJI will result in early implant failure with an untreated infection [7].

In order to maximise the accuracy of periprosthetic tissue culture, research has focused on numerous factors including types of samples [8-10] [11-13], culture methods [5, 14] [15] and methods of sampling [16]. The surgeon is currently advised to use a different set of instruments to minimise contamination with suspected infection and it is 
recommended that a minimum of three samples are taken during surgery [3].

Regarding the specific tissue type or anatomical location of the samples, the evidence base is lacking and associated guidance is vague. Tissue samples have been shown to have superior sensitivity and specificity compared to deep intra-operative swab samples in cases where tissue samples were selected in the areas that appeared most inflamed and infected [17]. Sampling of joint fluid through aspiration has variable results with a wide range of reported sensitivities (50-93\%) and specificities (82-97\%) [18]. It is acknowledged that superficial swabs are poor quality samples with low sensitivity and specificity [8]. Superficial wound fluid samples have also been shown to have poor accuracy in the diagnosis of PJI. Evidence suggests that in order to maximise the sensitivity and specificity of intra-operative specimen cultures at least five or six specimens should be obtained during surgery [19].

It has also been reported that adjunct investigations and patient factors can be used in combination to determine an individual's risk of having a PJI [20]. Similarly, a logistic regression model has also been designed to predict the likelihood of surgical debridement successfully treating PJI [21]. These predictive models can be used to support clinical decision making and as well as during patient counselling.

The primary aim of the current study was to examine the impact of anatomical site and tissue sample type in the diagnosis of hip and knee PJI. The secondary aim was to create a prediction model of PJI incorporating patient factors along with serum inflammatory markers and microbiology results, which have previously been used to predict the risk of PJI [22]. The null hypothesis was that the sensitivity and specificity of intra-operative samples are not affected by sample type or anatomical location.

\section{Materials and Methods}

\section{Method Design}

This was a retrospective cohort study investigating the effect of tissue type and anatomical location of intra-operative samples on the accuracy in the diagnosis of PJI. The standard for comparison was the clinical diagnosis made by the treating surgical team of an infected or non-infected prosthetic joint. A prediction model for PJI was then created using pre-operative patient factors in isolation and also in combination with intra-operative cultures. This study was conducted in collaboration with orthopaedic surgeons, microbiologists and diagnostic laboratory services at a tertiary centre.

\section{Identification of microbiology samples}

A consecutive cohort of all microbiology intra-operative periprosthetic samples taken from hips or knees between January 2010 and December 2016 was identified. The patient cohort was identified from the Laboratory Information Management System (LIMS) by using search tool Cognos [23] to identify all culture samples codes logged from patients who had been admitted to one of the centre's two orthopaedic wards at the time the sample was taken. For the seven year search period 4451 individual sample codes were identified $(2010 \mathrm{n}=510$, $2011 \mathrm{n}=551,2012 \mathrm{n}=585,2013 \mathrm{n}=713,2014 \mathrm{n}=770,2015$ $\mathrm{n}=789,2016 \mathrm{n}=533)$. The data search was performed by a laboratory technician and then crosschecked by an orthopaedic research fellow. Each sample code also had a corresponding sample label and final culture result with an isolated organism if positive. These codes were then cross referenced using a separate microbiology laboratory system, Laboratory Medicine Results, to identify the unique patient numbers linked to each sample. The data was then sorted according to unique patient identification number.

\section{Patient Cohort}

Figure 1 illustrates how the final cohort was formed. From the initial cohort of 4451 samples, 383 samples were removed after initial review as the sample label indicated the sample was not taken from either a hip or knee, and a further 608 samples were removed as they were not taken from a prosthetic hip or knee joint (i.e. were taken from a native joint). This left a remaining cohort of 3460 sample codes from 887 patients.

These 887 patient numbers were then entered into the centre's electronic records system, which contains information on laboratory results, radiological investigations, discharge letters and clinic letters. Combined with the microbiology sample information from LIMS each individual patient had data collected for: date of birth, sex, past medical history, orthopaedic history, pre-operative white blood cell (WBC) count, C-reactive protein (CRP) and erythrocyte sedimentation rate (ESR), type of surgery performed at time of sampling, type of sample and request information (i.e. site/tissue type/method of collection), culture results, organisms identified and sensitivities (if culture positive: all specimens that had grown a micro-organism and had sensitivities available were considered positive irrespective of any query regarding contamination), clinical diagnosis of PJI versus aseptic joint and subsequent management plans. Due to the retrospective design of the study data was not collected regarding when the specimens were collected in relation to the stage of the procedure 
and antibiotic administration, nor was information available regarding the surgeon's reasoning for selecting a particular sample type, for example whether the area looked clinically infected.

The final cohort of 3460 samples taken from 887 patients with 1076 patient-operation pairs provided the material to test the null hypothesis that sample tissue type or anatomical location would not affect the diagnostic reliability for PJI, using the clinical diagnosis of PJI versus aseptic joint as the standard for comparison.

\section{Statistical analysis}

Simple descriptive statistics were undertaken to calculate the sensitivity, specificity, positive predictive value (PPV) and negative predictive value (NPV) for intra-operative samples in diagnosing PJI. The sample culture test result (positive or negative) was compared against the clinical diagnosis of PJI (yes or no).

Statistical analysis was performed using Statistical Package for Social Sciences version 17.0 (SPSS Inc., Chicago, IL, USA). A Student's t-test and ANOVA were used to compare continuous variables between groups. Dichotomous variables were assessed using a Chi square test. A $p$ value of $<0.05$ was defined as significant.

A logistic regression model [24] was fitted using the glm (generalised linear model) function in R [25] to clinical diagnosis (gold standard) using the covariates: age, WBC count, revision, joint and sample tissue type, with and without the intra-operative sample culture result. Model selection was performed using the Bayesian Information Criterion (BIC) [26], and the best models with and without the culture results for prediction of PJI were compared. The success of predictions can be visualised using a Receiver Operating Characteristic (ROC) curve for the model which shows the relationship between false positive predictions and false negative predictions.

\section{Ethical statement}

The authors conducted a retrospective service evaluation, as such there was no additional patient contact and no requirement for formal ethical approval. The project was registered with the institutions audit department (registration number 7851) and was conducted in accordance with the Declaration of Helsinki and the guidelines for good clinical practice.

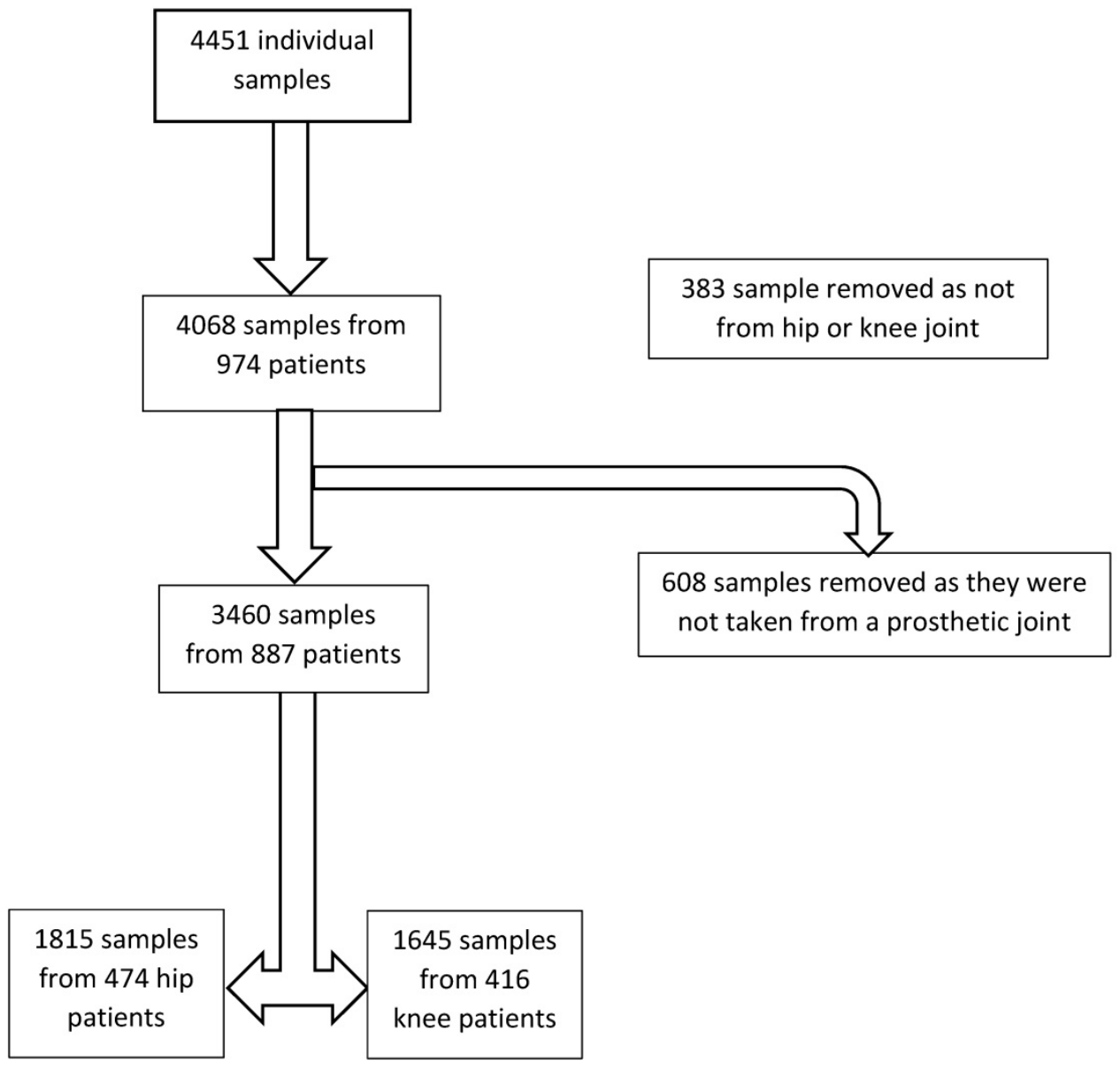

Figure 1: Flow chart showing development of final patient cohort from initial data set 


\section{Results}

The final cohort had 1815 samples taken during 557 operations from 474 patients undergoing hip surgery and 1645 samples taken during 519 operations from 416 patients undergoing knee surgery (three patients underwent both hip and knee surgery). The mean age was 68 years (range 24-94), with 493 female and 394 male. 314 patients had medical co-morbidities that could increase their risk of infection (diabetes, peripheral vascular disease, rheumatological conditions, vascular disease, renal failure, hepatic failure, chronic pulmonary disease [27]). Figure 2 shows the rate of occurrence of these co-morbidities within the cohort.

Table 1 shows the patient demographics per operation type. Total hip arthroplasty (THA) revision was the most common hip surgery $(\mathrm{N}=164)$ and total knee arthroplasty (TKA) revision was the most common knee surgery $(\mathrm{N}=204)$. Table 2 shows comparison between the patient cohorts of hip and knee surgery. There was a statistically significant difference $(p<0.05)$ between the two groups with regards to age, male:female ratio and proportion of patients with a clinical diagnosis of PJI. There was no statistically significant difference between the hip and knee surgery cohorts with regards to the proportion of patients with a medically increased risk of infection $(\mathrm{p}=0.1)$.

\section{Reliability of tissue type}

There were 3460 samples taken from the 887 patient-operation pairs. There were 1645 samples taken from knees and 1815 from hips. 1411 hip samples and 1025 knee samples were taken from patients without a clinical diagnosis of PJI. 404 hip samples and 620 knee samples were taken from patients with a clinical diagnosis of PJI. Table 3 shows the mean number of samples taken within the cohort. Figure 3 shows the variability in the number of samples taken within the cohort.

Table 4 shows the sensitivities, specificities, PPVs and NPVs for samples as per tissue type. Pus had the highest sensitivity $(83 \%)$ but the lowest specificity $(67 \%)$. The highest specificity was for synovium (93\%). Table 4 shows the diagnostic accuracy of samples as per the anatomical location. Hip capsule had the highest sensitivity (68\%) and there was $100 \%$ specificity for hip bursa. Knee femur and tibia also had high specificities $(90 \%$ and $89 \%$ respectively) but low sensitivities (32\% and 34\% respectively).

\section{Predictive model}

The cohort as a whole was then analysed to create a model for PJI using pre-operative factors: increased medical risk of infection, age, type of joint, pre-operative inflammatory markers (WBC count, CRP and ESR) and whether the operation was a revision surgery. The best fitting model included the covariates: WBC count, whether the operation was a revision, and the sample tissue type. Age did not improve the fit of the model. Revisions (single, first or second stage) decreased the probability of infection.

Adding culture results to the prediction model improved the fit considerably, and again comparison of the Bayesian information criterion (BIC) for the models gave a best model with WBC count, revision and sample tissue type and additionally, the culture result. All of these co-variants were significant with $\mathrm{p}<0.0001$.

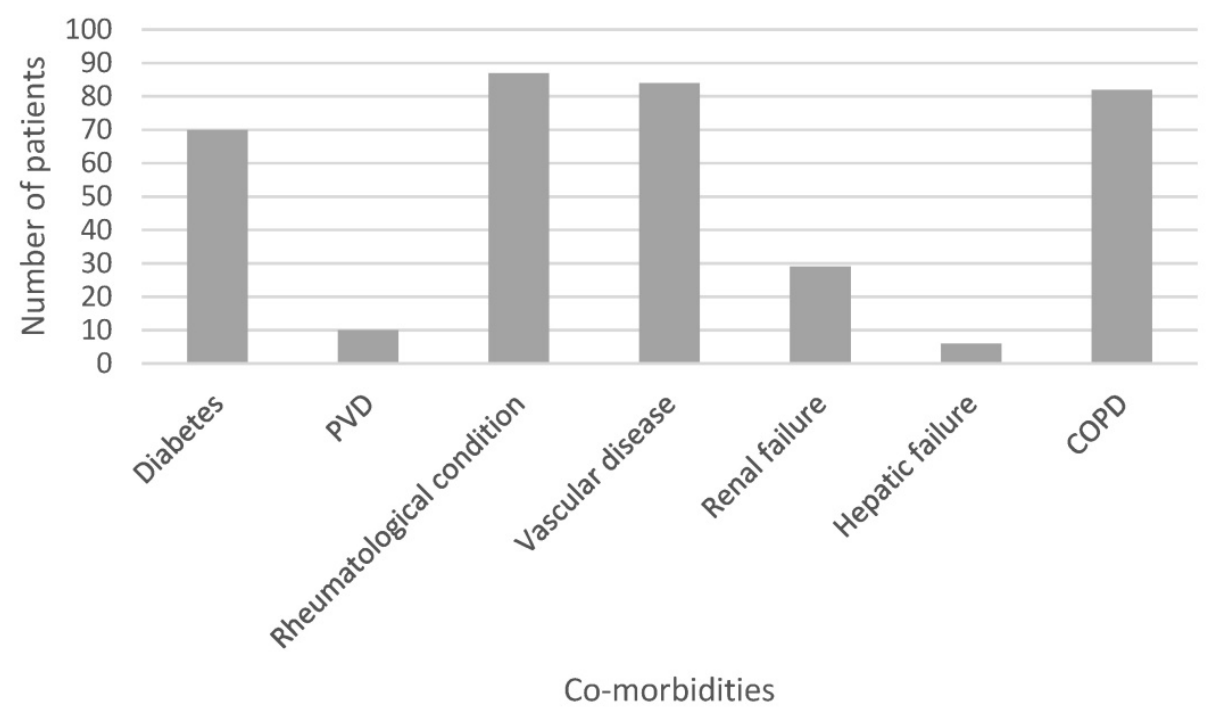

Figure 2: Bar chart showing the prevalence within the cohort of each co-morbidity associated with an increased risk of infection 
Table 1: The final patient cohort divided per operation

\begin{tabular}{|c|c|c|c|c|}
\hline & \multicolumn{4}{|l|}{ Patient demographics } \\
\hline & Average age (years) & Sex M:F & $\%$ of patients with medical co-morbidities & \% with clinical diagnosis of PJI \\
\hline \multicolumn{5}{|l|}{ HIP SURGERY } \\
\hline Primary total hip arthroplasty (THA) $(\mathrm{n}=75)$ & 65.3 & $33: 42$ & $45.3 \%$ & $6.7 \%$ \\
\hline THA revision (single stage) $(n=164)$ & 70.0 & 78:86 & $28.7 \%$ & $4.3 \%$ \\
\hline THA $1^{\text {st }}$ stage revision $(n=23)$ & 65.9 & $16: 7$ & $39.1 \%$ & $100 \%$ \\
\hline THA $2^{\text {nd }}$ stage revision $(\mathrm{n}=21)$ & 66.7 & $12: 9$ & $19.0 \%$ & $9.5 \%$ \\
\hline THA washout/debridement $(\mathrm{n}=31)$ & 71.2 & $16: 15$ & $32.3 \%$ & $71.0 \%$ \\
\hline THA aspiration $(n=9)$ & 66.6 & $3: 6$ & $22.2 \%$ & $44.4 \%$ \\
\hline Dynamic hip screw (DHS) converted to THA $(\mathrm{n}=22)$ & 74.3 & $2: 20$ & $63.6 \%$ & $0 \%$ \\
\hline Acetabulum revision $(n=138)$ & 68.5 & $45: 93$ & $26.1 \%$ & $2.2 \%$ \\
\hline Stem revision $(\mathrm{n}=11)$ & 71.1 & $6: 5$ & $45 \%$ & $9.1 \%$ \\
\hline Hemiarthroplasty revised to THA $(n=20)$ & 77.1 & $2: 18$ & $45 \%$ & $5 \%$ \\
\hline Hemiarthroplasty $(n=3)$ & 82.3 & $1: 2$ & $33.3 \%$ & $66.7 \%$ \\
\hline Resurfacing revised to THA $(n=23)$ & 52.6 & 9:14 & $30.4 \%$ & $0 \%$ \\
\hline Statistical difference $-{ }^{*}$ ANOVA ${ }^{* *}$ Chi squared test & $0^{*}$ & $0.0003^{\star * *}$ & $0.02184^{* *}$ & $0^{* *}$ \\
\hline All hip surgery $(\mathrm{n}=557)$ & 68.6 & 229:328 & $33.0 \%$ & $14.5 \%$ \\
\hline \multicolumn{5}{|l|}{ KNEE SURGERY } \\
\hline Primary total knee arthroplasty(TKA) $(n=51)$ & 66.8 & $27: 24$ & $45.1 \%$ & $0 \%$ \\
\hline TKA revision (single stage) $(\mathrm{n}=204)$ & 69.0 & 108:96 & $39.2 \%$ & $7.8 \%$ \\
\hline TKA $1^{\text {st }}$ stage revision $(n=66)$ & 65.9 & $44: 22$ & $41.0 \%$ & $100 \%$ \\
\hline TKA $2^{\text {nd }}$ stage revision $(\mathrm{n}=39)$ & 64.8 & $24: 15$ & $28.2 \%$ & $28.2 \%$ \\
\hline TKA washout/debridement $(n=55)$ & 62.0 & $29: 26$ & $43.6 \%$ & $61.8 \%$ \\
\hline TKA aspiration $(n=6)$ & 63.4 & $5: 1$ & $66.6 \%$ & $66.6 \%$ \\
\hline TKA patellar resurfacing $(n=60)$ & 66.5 & 19:41 & $31.7 \%$ & $1.7 \%$ \\
\hline Arthrodesis $(n=7)$ & 63.4 & $5: 2$ & $38.6 \%$ & $38.6 \%$ \\
\hline Arthrodesis revised to TKA $(n=2)$ & 60.6 & $1: 1$ & $0 \%$ & $0 \%$ \\
\hline Distal femoral replacement $(\mathrm{n}=2)$ & 80.8 & $2: 0$ & $0 \%$ & $0 \%$ \\
\hline Femoral component revision $(n=2)$ & 60.0 & $2: 0$ & $0 \%$ & $0 \%$ \\
\hline Patellofemoral replacement $(n=2)$ & 59.3 & $0: 2$ & $0 \%$ & $0 \%$ \\
\hline PF replacement revised to TKA $(n=12)$ & 61.1 & $2: 10$ & $33.3 \%$ & $0 \%$ \\
\hline Tibial revision $(\mathrm{n}=2)$ & 69.8 & $0: 2$ & $0 \%$ & $0 \%$ \\
\hline Uni revised to TKA $(n=9)$ & 67.5 & $4: 5$ & $22.2 \%$ & $0 \%$ \\
\hline Statistical difference - ANOVA* Chi squared test** & $0.00756^{*}$ & $0.001^{* *}$ & $0.47336^{* *}$ & $0^{* *}$ \\
\hline All knee surgery $(n=519)$ & 66.6 & 272:247 & $37.8 \%$ & $25.8 \%$ \\
\hline TOTAL ALL SURGERIES $(\mathrm{n}=1076)$ & 68.2 & $501: 575$ & $35.3 \%$ & $20.0 \%$ \\
\hline
\end{tabular}

Table 2: Patient demographics of hip and knee surgery cohorts

\begin{tabular}{|c|c|c|c|}
\hline & $\begin{array}{l}\text { Hip } \\
\text { surgery (n } \\
=557)\end{array}$ & $\begin{array}{l}\text { Knee } \\
\text { surgery }(n= \\
519)\end{array}$ & $\begin{array}{l}\text { Statistical difference } \\
\text { (Students T-test*, } \\
\text { Chi-squared test }^{* *} \text { ) }\end{array}$ \\
\hline Average age (years) & 68.6 & 66.6 & $\mathrm{p}=0.007^{*}$ \\
\hline Male:female ratio & $229: 328$ & $272: 247$ & $\mathrm{p}=0.0002^{* *}$ \\
\hline $\begin{array}{l}\% \text { with medical } \\
\text { co-morbidities increasing } \\
\text { risk of infection }\end{array}$ & $33.0 \%$ & $37.8 \%$ & $\mathrm{p}=0.104718^{* *}$ \\
\hline$\%$ with diagnosis of PJI & $\begin{array}{l}14.5 \% \\
(\mathrm{~N}=81)\end{array}$ & $\begin{array}{l}25.8 \% \\
(\mathrm{~N}=132)\end{array}$ & $\mathrm{p}<0.001^{*}$ \\
\hline
\end{tabular}

Table 3: Number of intra-operative samples taken from the study cohort

\begin{tabular}{llll}
\hline & $\begin{array}{l}\text { Total } \\
\text { cohort }\end{array}$ & $\begin{array}{l}\text { Knee } \\
\text { cohort }\end{array}$ & $\begin{array}{l}\text { Hip } \\
\text { cohort }\end{array}$ \\
\hline Mean number of samples (range) & $3.93(1-50)$ & $3.99(1-50)$ & $3.88(1-25)$ \\
Mean number of tissue types sampled (range) & $1.98(1-6)$ & $1.95(1-6)$ & $2.01(1-5)$ \\
$\begin{array}{l}\text { Mean number of anatomical locations sampled } \\
\text { (range) }\end{array}$ & $1.60(1-6)$ & $1.45(1-6)$ & $1.75(1-5)$ \\
\hline
\end{tabular}

Figure 4 shows the ROC curve for the PJI model. Using the results for tissue alone the false positive rate clustered around $10 \%$. To create a model with a superior false positive rate i.e. $5 \%$, the true positive rates of the model then decreased. Therefore, it appeared that no model is possible that produces improved false positive rates without compromising the true positive rates.

Table 4: Diagnostic accuracy divided by tissue type and anatomical location

\begin{tabular}{|c|c|c|c|c|c|}
\hline & $\begin{array}{l}\text { Number of } \\
\text { samples (number } \\
\text { of patients) }\end{array}$ & Sensitivity & Specificity & PPV & NPV \\
\hline \multicolumn{6}{|l|}{ Tissue type } \\
\hline Bone & $90(68)$ & $45 \%(30-61)$ & $85 \%(71-94)$ & $74 \%$ & $62 \%$ \\
\hline Capsule & $185(113)$ & $61 \%(41-78)$ & $90 \%(85-95)$ & $53 \%$ & $93 \%$ \\
\hline Fluid & $928(660)$ & $39 \%(33-46)$ & $92 \%(90-94)$ & $64 \%$ & $82 \%$ \\
\hline Membrane & $207(140)$ & $43 \%(26-61)$ & $86 \%(80-91)$ & $38 \%$ & $88 \%$ \\
\hline Pus & $15(13)$ & $83 \%(52-98)$ & $67 \%(9-99)$ & $91 \%$ & $50 \%$ \\
\hline Synovium & $131(87)$ & $41 \%(25-59)$ & $93 \%(86-97)$ & $67 \%$ & $82 \%$ \\
\hline Tissue & $1886(654)$ & $50 \%(46-54)$ & $88 \%(86-90)$ & $68 \%$ & $78 \%$ \\
\hline \multicolumn{6}{|c|}{ Anatomical location } \\
\hline All hip & $1815(473)$ & $58 \%(53-63)$ & $89 \%(88-91)$ & $61 \%$ & $88 \%$ \\
\hline Hip acetabulum & 337 (188) & $58 \%(44-71)$ & $91 \%(88-94)$ & $58 \%$ & $91 \%$ \\
\hline Hip bursa & $13(12)$ & $75 \%(19-99)$ & $100 \%(66-100)$ & $100 \%$ & $90 \%$ \\
\hline Hip capsule & $173(101)$ & $68 \%(48-84)$ & $90 \%(84-95)$ & $58 \%$ & $94 \%$ \\
\hline Hip femur & $167(113)$ & $56 \%(41-71)$ & $88 \%(81-93)$ & $66 \%$ & $83 \%$ \\
\hline All knee & 1645 (417) & $41 \%(37-45)$ & $90 \%(88-92)$ & $71 \%$ & $71 \%$ \\
\hline Knee femur & $120(76)$ & $35 \%(22-50)$ & $90 \%(80-96)$ & $72 \%$ & $65 \%$ \\
\hline Knee patella & $34(26)$ & $42 \%(15-72)$ & $77 \%(55-92)$ & $50 \%$ & $71 \%$ \\
\hline Knee tibia & $92(72)$ & $34 \%(19-52)$ & $89 \%(78-96)$ & $67 \%$ & $69 \%$ \\
\hline
\end{tabular}

Uncertainty of estimates in brackets 


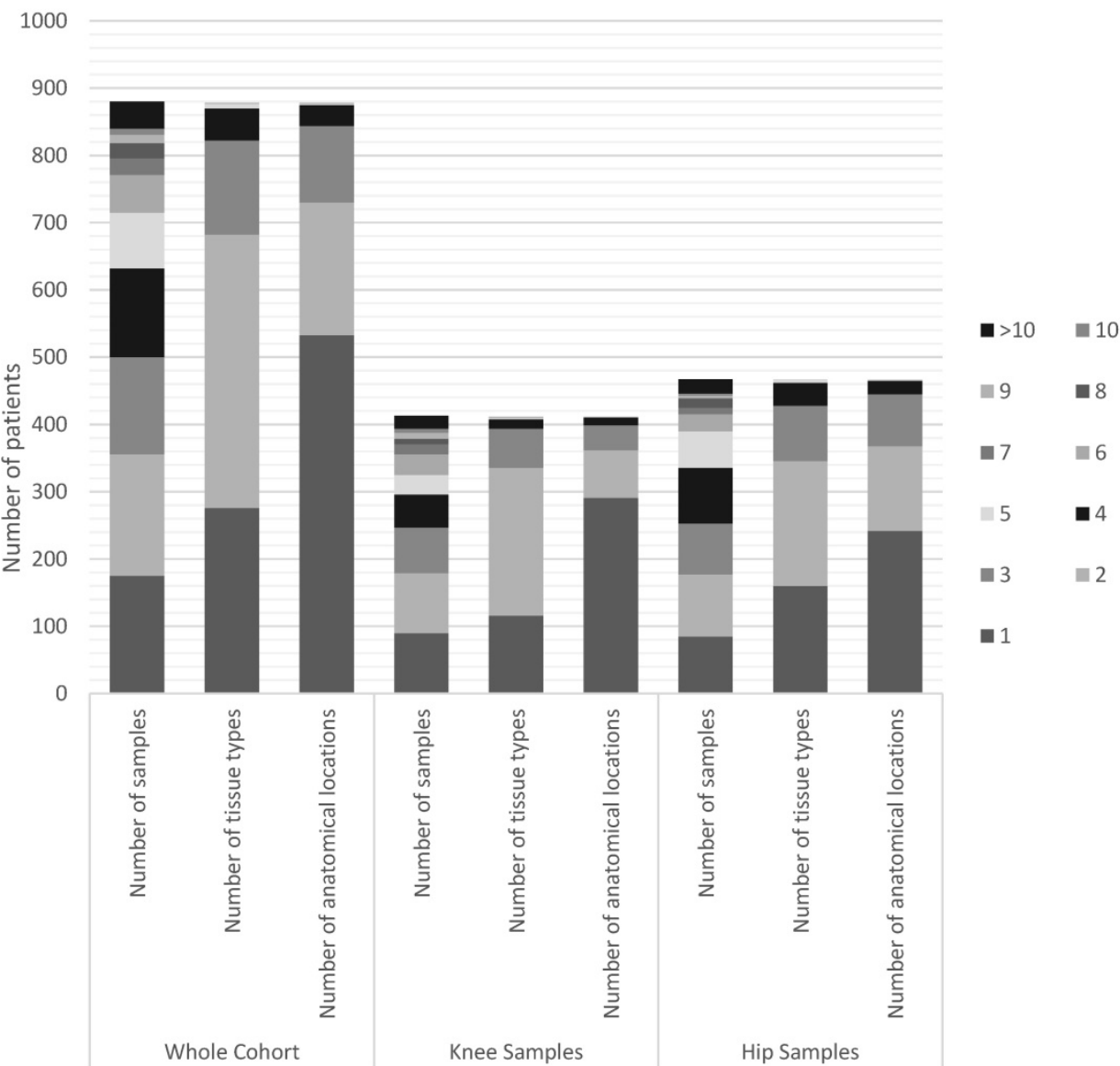

Figure 3: Distribution of the number and variability of samples taken intra-operatively across the cohort

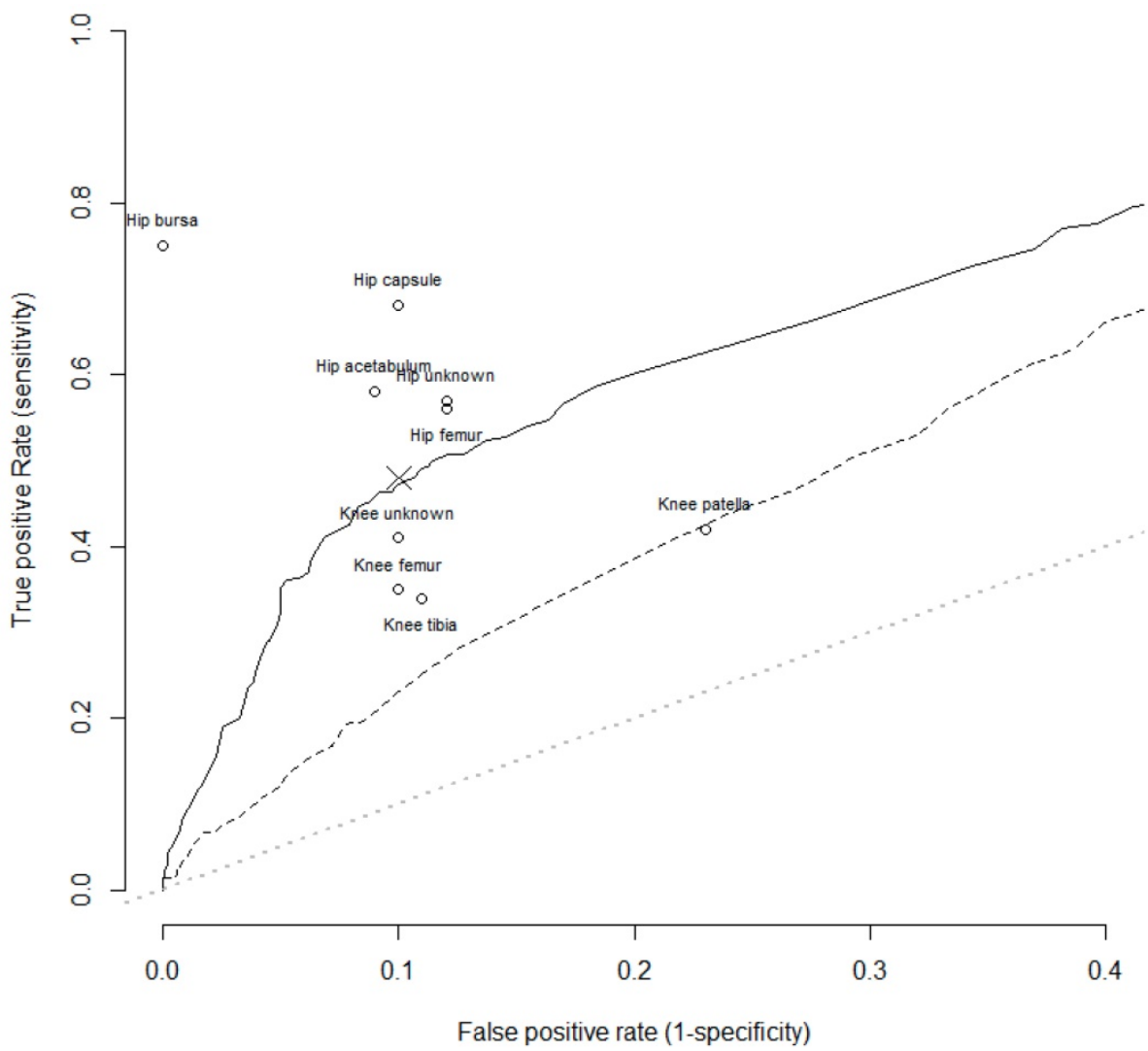

Figure 4: ROC curve analysis of PJI predictor model without culture information (dashed line, middle line) and using culture information (solid line). The lower dotted line indicates a test with no prognostic power. Circles are from the raw data for tissue types, the cross gives the overall sensitivity and specificity. 


\section{Discussion}

The key finding of this investigation is that there is a variation in diagnostic accuracy between intra-operative samples with differing tissue types and anatomical location. However, no single sample type had diagnostic accuracy comparable to previously reported rates of sensitivity, specificity and predictive values of intra-operative culture samples considered collectively [17], thereby highlighting the necessity for sampling from multiple sites and tissue types in the diagnosis of PJI. It was also shown that by factoring in patients' pre-operative WBC count, type of surgery and culture result along with sample tissue type the best possible predictive model of PJI was created.

\section{Limitations}

One limitation of this study is the retrospective design, so data could not be collected on the surgeons' decision making in selecting the samples types and sites, nor could the accuracy of the sample labelling be controlled. However, the dataset was collected independent of any specific hypothesis therefore excluding the potential for bias. Data was not collected regarding when the specimens were collected in relation to the stage of the procedure and antibiotic administration. Trampuz et al.[28] demonstrated that any use of antibiotics in the two weeks before obtaining culture samples was associated with a lower yield of cultures from sonication samples obtained from hip and knee prostheses. This could therefore, have impacted upon the culture results of current studies samples. Within the study centre antibiotics are routinely held until after culture samples are taken, although data regarding compliance within the protocol or pre-operative antibiotic administration in the community were not available. The current study also included samples taken from patients during second stage revision surgeries who had previously had antibiotic-loaded cement spacers in situ and systemic antibiotics. It is possible persistent antibiotic elution may yield false negative culture results [29]. However, it has also been shown that new infective organisms can be identified between explantation and re-implantation [30,31] so the authors felt it important that these samples were included in the analysis as a new PJI pathogen can be diagnosed at the second stage surgery.

It could also be argued that the choice of the documented clinical diagnosis of PJI as the gold standard for comparison is a limitation. The recorded diagnosis of PJI versus an aseptic joint was collected in the dataset based solely on the documentation of the treating clinical team, without reviewing or revising the basis of their diagnostic decision making. The majority of the current literature uses intra-operative culture results as one of the criterion for their gold standard but as that was the variable the current study was assessing a different standard was chosen. Furthermore, using culture results as a gold standard does have its limitations as false negative culture results have been reported with a frequency that ranges from $7-23 \%$ [32]. The specific causative micro-organism can also have an influence on culture specificity, with virulent organisms such as Staphylococcus aureus growing easily [5] whereas atypical organisms do not grown on routine media and organisms such as Propionibacterium acnes need one to two weeks for isolation [33-36]. There are accepted diagnostic criteria available that could have been selected as a gold standard, such as the Musculoskeletal Infection Society's (MSIS) Workgroup criteria [5]. However, this was not used in the current study as the majority of patients did not have all investigations completed to use this criteria. Furthermore, Honkanen et al.[37] have suggested that true PJI cases could be missed by the MSIS criteria as a result of exclusion of clinical decision making.

The majority of samples analysed in the current study had been taken from patient without a clinical diagnosis of PJI. The majority of cases also had one to three samples taken, rather than the recommended five or six samples [19]. This raises the question of the clinical reasoning behind taking samples if cases were clearly of a non-infected nature and the amount of resources being spent on these tests. It has previously been reported that up to $£ 23900000$ was spent within the NHS on unnecessary respiratory microbiological testing [38] and this may reflect a similar issue within orthopaedics. 129 samples were taken from primary hip replacements and 87 samples were taken from primary knee arthroplasty which would be presumed to be non-infected however, there were five cases of PJI cases in primary total hip arthroplasties although unfortunately further details regarding these cases were not available.

\section{Diagnostic accuracy of samples per tissue type and location}

The current study found fluid samples to have a low sensitivity but high specificity, which has previously been reported by Gallo et al.[39] Synovial tissue samples in the current study also had low sensitivity which is comparable to the findings of Cross et al.[40] and questions the utility of pre-operative synovial biopsies. Collectively intra-operative cultures in this study had much lower sensitivity than the results reported by Fink et al.[16] 
for tissue biopsy results, however, they were assessing pre-operative percutaneous tissue samples rather than those taken intra-operatively.

The tissue type and anatomical location of samples has been shown by the current study to have varying predictive values for PJI. For tissue type, pus had the highest PPV although there was a small sample size for this group. Capsule had the most superior NPV. For hips, bursa samples had high PPV and NPV. It is recommended that tests with high NPV be used to screen for infection and those with high PPV be used to confirm a diagnosis of PJI [41], and therefore the findings of the current study could guide treating surgeons on which sample types should be taken depending on their level of clinical suspicion of PJI. The variation in diagnostic accuracy across all sample types also highlights the need for multi-site sampling in the diagnosis of PJI. This supports the MSIS criteria that recommends a minimum of three intra-operative samples are taken [3].

\section{Predictive model for PJI}

Using co-variates to create a predictive model for PJI showed pre-operative WBC count, type of surgery (revision versus non-revision) and sample tissue joint type (knee or hip) to be the best fit. Patients with non-revision (type of surgery) and WBC count $>10 \mathrm{x}$ 109 cells per litre and tissue from knee joints were found to be at increased risk of a PJI diagnosis. The finding that knee prostheses have an increased risk of PJI correlates with Pulido et al.[42] who also reported a higher incidence of PJI in knee replacements than for hip replacements. Serum WBC count rises in response to infection, therefore would be expected to be raised in PJI. However, its use as a single diagnostic marker of PJI has been discouraged due to its poor sensitivity and specificity [43,44]. Revision surgery decreasing the risk of a PJI diagnosis could be explained by the routine surgical practice of taking intra-operative samples for all revision surgeries, even those with a very low clinical suspicion of infection. Therefore, if only an isolated sample was culture positive but there was no clinical suggestion of infection then it can still be considered an aseptic joint. Whereas, for primary surgery samples are not routinely taken, therefore in the cases that do have cultures sent it may be due to pre-existing clinical suspicion of infection. When using these factors in combination with intra-operative culture results for predicting PJI the sensitivity improved but the specificity decreased. Therefore, in order to increase the sensitivity of a PJI diagnosis it is useful to consider other diagnostic modalities, such as pre-operative inflammatory markers, patient and surgical factors.
The current study has demonstrated a variation in sensitivity, specificity and predictive values according to intra-operative sample type. Due to the variation in predictive values, the operating surgeons may wish to alter which intra-operative sample types are selected depending on their level of clinical suspicion, as well as considering pre-operative patient and joint variants in the diagnostic process.

\section{Conclusion}

In conclusion, this study analysed intra-operative samples collected irrespective of type and presence of infection and irrespective of the surgeon's reasoning for selecting a particular sample type. Whilst this did demonstrated variation in diagnostic accuracy depending on the tissue type and anatomical location of intra-operative culture samples taken to diagnose PJI, no individual tissue or anatomical type had superior accuracy compared to all samples used collectively. However, the diagnostic reliability was increased when pre-operative inflammatory markers and surgical factors (type or joint and primary or revision arthroplasty) were incorporated into a predictive model for PJI.

\section{Abbreviations}

PJI: Periprosthetic joint infection

LIMS: Laboratory information management system

WBC: White blood cell

CRP: C-reactive protein

ESR: Erythrocyte sedimentation rate

PPV: Positive predictive value

NPV: Negative predictive value

SPSS: Statistical package for social sciences

BIC: Bayesian information criterion

ROC: Receiver operating characteristic

THA: Total hip arthroplasty

TKA: Total knee arthroplasty

MSIS: Musculoskeletal infection society

\section{Author Contributions}

LCW performed the investigation, data curation and writing of the original draft manuscript and re-editing. NDC reviewed and edited the manuscript. IW performed the formal data analysis. $\mathrm{MH}$ supervised the project as well as reviewing the manuscript. JS helped devise the metholodology and data curation by allowing access to the necessary software. DJD devised the concept of the project and provided supervision throughout as well as reviewing and editing the manuscript.

\section{Competing Interests}

Authors LCW, NDC, IW and JS declare no competing interests. 
Author $\mathrm{MH}$ has instructed on ZimmerBiomet courses.

Author DJD has received funding from Stryker for separate projects on knee biomechanics.

\section{References}

1. Munemoto M, Inagaki Y, Tanaka Y, Grammatopoulos G, Athanasou NA. Quantification of neutrophil polymorphs in infected and noninfected second-stage revision hip arthroplasties. Hip Int. 2016; 26: 327-30.

2. Springer BD, Cahue S, Etkin CD, Lewallen DG, McGrory BJ. Infection burden in total hip and knee arthroplasties: an international registry-based perspective. Arthroplasty today. 2017; 3: 137-40.

3. Gehrke T, Parvizi J. Proceedings of the international consensus meeting on periprosthetic joint infection. J Arthroplasty. 2014; 29: 4 .

4. Osmon DR, Berbari EF, Berendt AR, Lew D, Zimmerli W, Steckelberg JM, et al. Diagnosis and management of prosthetic joint infection: clinical practice guidelines by the Infectious Diseases Society of America. Clin Infect Dis. 2012; 56: e1-e25.

5. Parvizi J, Zmistowski B, Berbari EF, Bauer TW, Springer BD, Della Valle CJ, et al. New definition for periprosthetic joint infection: from the Workgroup of the Musculoskeletal Infection Society. Clin Orthop. 2011; 469: 2992.

6. Gollwitzer H, Dombrowski Y, Prodinger PM, Peric M, Summer B, Hapfelmeier A, et al. Antimicrobial peptides and proinflammatory cytokines in periprosthetic joint infection. J Bone Joint Surg. 2013; 95: 644-51.

7. Squire MW, Valle CJD, Parvizi J. Preoperative diagnosis of periprosthetic joint infection: role of aspiration. Am J Roent. 2011; 196: 875-9.

8. Aggarwal VK, Higuera C, Deirmengian G, Parvizi J, Austin MS. Swab cultures are not as effective as tissue cultures for diagnosis of periprosthetic joint infection. Clin Orthop. 2013; 471: 3196-203.

9. Della Valle C, Parvizi J, Bauer TW, DiCesare PE, Evans RP, Segreti J, et al. American Academy of Orthopaedic Surgeons clinical practice guideline on: the diagnosis of periprosthetic joint infections of the hip and knee. J Bone Joint Surg. 2011; 93: 1355-7.

10. Tetreault MW, Wetters NG, Aggarwal VK, Moric M, Segreti J, Huddleston JI, et al. Should draining wounds and sinuses associated with hip and knee arthroplasties be cultured? J Arthroplasty. 2013; 28: 133-6.

11. Parvizi J, Ghanem E, Sharkey P, Aggarwal A, Burnett RSJ, Barrack RL. Diagnosis of infected total knee: findings of a multicenter database. Clin Orthop. 2008; 466: 2628-33.

12. Schinsky MF, Della Valle CJ, Sporer SM, Paprosky WG. Perioperative testing for joint infection in patients undergoing revision total hip arthroplasty. J Bone Joint Surg. 2008; 90: 1869-75.

13. Della Valle CJ, Sporer SM, Jacobs JJ, Berger RA, Rosenberg AG, Paprosky WG. Preoperative testing for sepsis before revision total knee arthroplasty. J Arthroplasty. 2007; 22: 90-3.

14. Smith EB, Cai J, Wynne R, Maltenfort M, Good RP. Performance characteristics of broth-only cultures after revision total joint arthroplasty. Clin Orthop. 2014; 472: $3285-90$

15. Geller JA, MacCallum KP, Murtaugh TS, Patrick DA, Liabaud B, Jonna VK. Prospective comparison of blood culture bottles and conventional swabs for microbial identification of suspected periprosthetic joint infection. J Arthroplasty. 2016; 31: 1779-83.

16. Fink B, Gebhard A, Fuerst M, Berger I, Schäfer P. High diagnostic value of synovial biopsy in periprosthetic joint infection of the hip. Clin Orthop. 2013; 471: 956-64

17. Spangehl MJ, Masri BA, O'connell JX, Duncan CP. Prospective analysis of preoperative and intraoperative investigations for the diagnosis of infection at the sites of two hundred and two revision total hip arthroplasties. J Bone Joint Surg. 1999; 81: 672-83.

18. Ali F, Wilkinson JM, Cooper JR, Kerry RM, Hamer AJ, Norman P, et al. Accuracy of joint aspiration for the preoperative diagnosis of infection in total hip arthroplasty. J Arthroplasty. 2006; 21: 221-6.

19. Atkins BL, Athanasou N, Deeks JJ, Crook DW, Simpson H, Peto TE, et al. Prospective evaluation of criteria for microbiological diagnosis of prosthetic-joint infection at revision arthroplasty. J Clin Microbiol. 1998; 36: 2932-9.

20. Berbari EF, Osmon DR, Lahr B, Eckel-Passow JE, Tsaras G, Hanssen AD, et al. The Mayo prosthetic joint infection risk score: implication for surgical site infection reporting and risk stratification. Infect Control Hosp Epidemiol. 2012; 33: 774-81.

21. Buller LT, Sabry FY, Easton RW, Klika AK, Barsoum WK. The preoperative prediction of success following irrigation and debridement with polyethylene exchange for hip and knee prosthetic joint infections. J Arthroplasty. 2012; 27: 857-64. e4.

22. Bedair H, Ting N, Jacovides C, Saxena A, Moric M, Parvizi J, et al. The Mark Coventry Award: diagnosis of early postoperative TKA infection using synovial fluid analysis. Clin Orthop. 2011; 469: 34-40.

23. Ghosh S, Sharma N, Benevenuto F, Ganguly N, Gummadi K. Cognos: crowdsourcing search for topic experts in microblogs. Proceedings of the 35th international ACM SIGIR conference on Research and development in information retrieval: ACM; 2012. p. 575-90.

24. Veneables W, Ripley B. Modern applied statistics with S-PLUS. 2da. 1997.
25. Team RC. R: A language and environment for statistical computing. $R$ Foundation for Statistical Computing. 2014. 2018

26. Schwarz G. Estimating thedimension of a model. Annals ofStatistics, 6 (2), 461-464. 1978

27. Bozic KJ, Lau E, Kurtz S, Ong K, Berry DJ. Patient-related risk factors for postoperative mortality and periprosthetic joint infection in medicare patients undergoing TKA. Clin Orthop. 2012; 470: 130-7.

28. Trampuz A, Piper KE, Jacobson MJ, Hanssen AD, Unni KK, Osmon DR, et al. Sonication of removed hip and knee prostheses for diagnosis of infection. N Engl J Med. 2007; 357: 654-63.

29. Newman JM, George J, Klika AK, Hatem SF, Barsoum WK, North WT, et al. What is the diagnostic accuracy of aspirations performed on hips with antibiotic cement spacers? Clin Orthop. 2017; 475: 204-11.

30. Hoell S, Moeller A, Gosheger G, Hardes I, Dieckmann R, Schulz D. Two-stage revision arthroplasty for periprosthetic joint infections: what is the value of cultures and white cell count in synovial fluid and CRP in serum before second stage reimplantation? Arch Orthop Trauma Surg. 2016; 136: 447-52.

31. Cabo J, Euba G, Saborido A, González-Panisello M, Domínguez MA, Agulló $\mathrm{JL}$, et al. Clinical outcome and microbiological findings using antibiotic-loaded spacers in two-stage revision of prosthetic joint infections. J Infect. 2011; 63: 23-31.

32. Choi H-R, Kwon Y-M, Freiberg AA, Nelson SB, Malchau H. Periprosthetic joint infection with negative culture results: clinical characteristics and treatment outcome. J Arthroplasty. 2013; 28: 899-903.

33. Neogi DS, Kumar A, Yadav CS, Singh S. Delayed periprosthetic tuberculosis after total knee replacement: is conservative treatment possible? Acta Orthop Belg. 2009; 75: 136 .

34. Larson AN, Razonable RR, Hanssen AD. Case report: Capnocytophaga canimorsus a novel pathogen for joint arthroplasty. Clin Orthop. 2009; 467: 1634-8.

35. Zappe B, Graf S, Ochsner PE, Zimmerli W, Sendi P. Propionibacterium spp. in prosthetic joint infections: a diagnostic challenge. Arch Orthop Trauma Surg. 2008; 128: 1039-46.

36. Zimmerli W, Trampuz A, Ochsner PE. Prosthetic-joint infections. N Engl J Med. 2004; 351: 1645-54.

37. Honkanen M, Jämsen E, Karppelin M, Huttunen R, Lyytikäinen O, Syrjänen J. Concordance between the old and new diagnostic criteria for periprosthetic joint infection. Infection. 2017; 45: 637-43.

38. Abdi Y. The appropriateness of clinical microbiology laboratory investigations: a retrospective study of the cost and clinical relevance of specimen management and processing: University of Portsmouth; 2011.

39. Gallo J, Kolar M, Dendis M, Loveckova Y, Sauer P, Zapletalova J, et al. Culture and PCR analysis of joint fluid in the diagnosis of prosthetic joint infection. Microbiologica-Quarterly Journal of Microbiological Sciences. 2008; 31: 97-104.

40. Cross MC, Kransdorf MJ, Chivers FS, Lorans R, Roberts CC, Schwartz AJ, et al. Utility of percutaneous joint aspiration and synovial biopsy in identifying culture-positive infected hip arthroplasty. Skeletal Radiol. 2014; 43: 165-8.

41. Parvizi J, Ghanem E, Menashe S, Barrack RL, Bauer TW. Periprosthetic infection: what are the diagnostic challenges? J Bone Joint Surg. 2006; 88: 138-47.

42. Pulido L, Ghanem E, Joshi A, Purtill JJ, Parvizi J. Periprosthetic joint infection: the incidence, timing, and predisposing factors. Clin Orthop. 2008; 466: 1710-5.

43. Berbari E, Mabry T, Tsaras G, Spangehl M, Erwin PJ, Murad MH, et al. Inflammatory blood laboratory levels as markers of prosthetic joint infection: a systematic review and meta-analysis. J Bone Joint Surg. 2010; 92: 2102-9.

44. Toossi N, Adeli B, Rasouli MR, Huang R, Parvizi J. Serum white blood cell count and differential do not have a role in the diagnosis of periprosthetic joint infection. J Arthroplasty. 2012; 27: 51-4. e1. 